\title{
Simulation of the Three-Dimensional Flow of Blood Using a Shear-Thinning Viscoelastic Fluid Model
}

\author{
T. Bodnár ${ }^{1}$, K.R. Rajagopal ${ }^{2}$ and A. Sequeira ${ }^{3}$ * \\ ${ }^{1}$ Department of Technical Mathematics, Faculty of Mechanical Engineering \\ Czech Technical University, Náměstí 13, 12135 Prague 2, Czech Republic \\ ${ }^{2}$ Department of Mechanical Engineering, Texas A \& M University \\ College Station, TX 77843-3123, USA \\ ${ }^{3}$ Department of Mathematics and CEMAT/IST, Instituto Superior Técnico \\ Technical University of Lisbon, Av. Rovisco Pais 1, 1049-001 Lisboa, Portugal
}

\begin{abstract}
This paper is concerned with the numerical simulation of a thermodynamically compatible viscoelastic shear-thinning fluid model, particularly well suited to describe the rheological response of blood, under physiological conditions. Numerical simulations are performed in two idealized three-dimensional geometries, a stenosis and a curved vessel, to investigate the combined effects of flow inertia, viscosity and viscoelasticity in these geometries. The aim of this work is to provide new insights into the modeling and simulation of homogeneous rheological models for blood and a basis for further developments in modeling and prediction.
\end{abstract}

Key words: blood rheology, viscoelasticity, stenosis, curved vessel, secondary flows AMS subject classification: 76A10, 76M12, $76 \mathrm{Z} 05$

\section{Introduction}

Blood is a complex mixture of several formed elements, red blood cells (RBCs or erythrocytes), white blood cells (WBCs or leukocytes) and platelets (thrombocytes), in an aqueous polymeric and ionic solution, the plasma, containing electrolytes, organic molecules and numerous proteins. Despite being such a complicated mixture, it can be modeled in sufficiently large blood vessels as

\footnotetext{
*Corresponding author. E-mail: adelia.sequeira@math.ist.utl.pt
} 
a single constituent fluid and, depending on the size of the blood vessels and the flow behvior, it is approximated as a Navier-Stokes fluid or as a non-Newtonian fluid.

The presence of the formed elements in blood leads to some significant and fascinating changes in its rheological properties and more experiments need to be performed, at the scale of the red blood cells, to extend the existing models by including microstructural aspects of blood. Reliable measurements of velocity and shear stress, as well as interactions between the cellular components of blood and plasma are essential to develop these microstructural models, appropriate in smaller vessels in which the cell and lumen sizes are comparable.

This paper concerns the flow of blood in vessels where it needs to be described by a nonNewtonian fluid model. There is a large body of literature confirming that blood shear thins due to the aggregation of the red blood cells, and the formation of rouleau 3D microstructures at low shear rates and their deformability at high shear $([15,16,17,18,19])$. There is also experimental evidence that supports the fact that blood is capable of stress relaxation, see e.g. Thurston [30], Quemada [27], Chien et al. [20]. Evans and Hochmuth [21] have found that the red blood cell membrane, which is a component of blood, exhibits stress relaxation. Moreover, the experimental results of Thurston [31] imply that the relaxation time depends on the shear rate. In view of the available experimental evidence, it is reasonable to develop a non-Newtonian fluid model for blood that is capable of shear-thinning and stress relaxation, with the relaxation time depending on the shear rate. To date, very little is known concerning the response of such fluids.

Rajagopal and Srinivasa [28] have developed a thermodynamic framework within which one could systematically develop rate type models to describe the viscoelastic response of fluids. The framework is general enough to develop models wherein the relaxation time depends on the shear rate. All that the thermodynamic framework requires is a knowledge of how the material stores energy and how it produces entropy. The models that are developed, automatically satisfy the second law of thermodynamics. In fact, the framework requires the rate of entropy production to be non-negative. Moreover, from amongst the class of admissible constitutive relations meeting the condition that the rate of entropy production is non-negative, this theory also requires that the model should maximize the rate of entropy production. Based on [28], Anand et al. [1] introduced a model that is suitable for describing the response characteristics of blood. This model contains the Oldroyd-B model as a special sub-class.

The theory developed by Rajagopal and Srinivasa [28] makes use of the fact that the natural configuration of the material changes as the body produces entropy, and also that one can unload from the current configuration of the body to its natural configuration through an instantaneous elastic response. As blood is modeled as a viscoelastic fluid capable of instantaneous elastic response, the framework developed in [28] is particularly well suited to develop a model for blood. This framework needs the specification of how the body stores and dissipates energy. The way in which the body stores energy is usually provided by associating a specific Helmholtz potential with the body, and how the body dissipates energy is given by a rate of dissipation function. However, as shown recently in [29], not all viscoelastic fluids can be described within that earlier framework. In fact, there are (rate type) viscoelastic fluids that cannot have a specific Helmholtz potential associated with them. For certain viscoelastic fluids one cannot associate a specific Helmholtz potential but only a Gibbs potential. Rate type fluids stemming from a Gibbs potential approach might be 
useful in describing the response of blood. In this paper we shall not pursue such models but restrict our attention to the model developed by Anand et al. [1] that has proven to be successful in describing the response of blood.

Anand et al. [2], [3], [4] have also studied the problem of the formation and lysis of blood clots, and more recently the problem of ATIII and protein C deficiency [5] within the context of the above model. Due to the complexity of the model, their studies are based on simple geometries and flow assumptions so that the problem under consideration can be reduced to a simple system of equations in one spatial dimension. However, if the model is to be used to study realistic problems with relevance to biomedicine, it is necessary to consider blood flow in more complex geometries than those studied in the above referenced papers, and to perform three-dimensional simulations. We address this issue in the present work. We consider the model described in [1] and perform a numerical investigation of the combined effects of inertia, viscosity and viscoelasticity of the fluid in two idealized three-dimensional geometries, a stenosis and a curved vessel. These combined effects are observed by comparing the results of the axial velocity, radial velocity and pressure contours for the new model with those obtained for classical blood flow models, namely the Newtonian and the generalized (shear-thinning) Oldroyd-B models, as previously done in [10, $13,14]$ (see also e.g. [24, 26, 23, 8]).

\section{The mathematical model}

\subsection{Kinematics}

In this section we provide the minimum kinematical definitions that will make this paper selfcontained. In order to understand the model that is being used in this work, it is necessary to define some kinematical quantities. The notation introduced in Rajagopal and Srinivasa [28] is used here. Let $\boldsymbol{X} \in \kappa_{R}$ denote a material point in the reference configuration $\kappa_{R}$ of the body $\mathcal{B}$ (see Fig. 1), and let $\boldsymbol{x} \in \kappa_{t}$ denote the same material point in the current configuration $\kappa_{t}$ at time $t$. Let $\kappa_{p(t)}$ denote the natural configuration that can be accessed by instantaneously unloading the body which is at the current configuration. As the body deforms, the underlying natural configuration $\kappa_{p(t)}$ changes. By the motion of a body we mean a one to one mapping $\chi(\boldsymbol{X}, t)$, that assigns to each point $\boldsymbol{X} \in \kappa_{R}$ a point $\boldsymbol{x} \in \kappa_{t}$, for each $t$, i. e.,

$$
\chi: \kappa_{R} \times \mathbb{R} \rightarrow \mathcal{E}, \quad \boldsymbol{x}:=\chi(\boldsymbol{X}, t) .
$$

We will assume that the motion is invertible and sufficiently smooth, in such a way that all derivatives that are taken make sense.

The deformation gradient $\boldsymbol{F}_{\kappa_{R}}$ is defined through

$$
\boldsymbol{F}_{\kappa_{R}}:=\frac{\partial \chi}{\partial \boldsymbol{X}}
$$

and the left and right Cauchy-Green stretch tensors $\boldsymbol{B}_{\kappa_{R}}$ and $\boldsymbol{C}_{\kappa_{R}}$ are defined through

$$
\boldsymbol{B}_{\kappa_{R}}:=\boldsymbol{F}_{\kappa_{R}} \boldsymbol{F}_{\kappa_{R}}^{T}, \quad \boldsymbol{C}_{\kappa_{R}}:=\boldsymbol{F}_{\kappa_{R}}^{T} \boldsymbol{F}_{\kappa_{R}},
$$




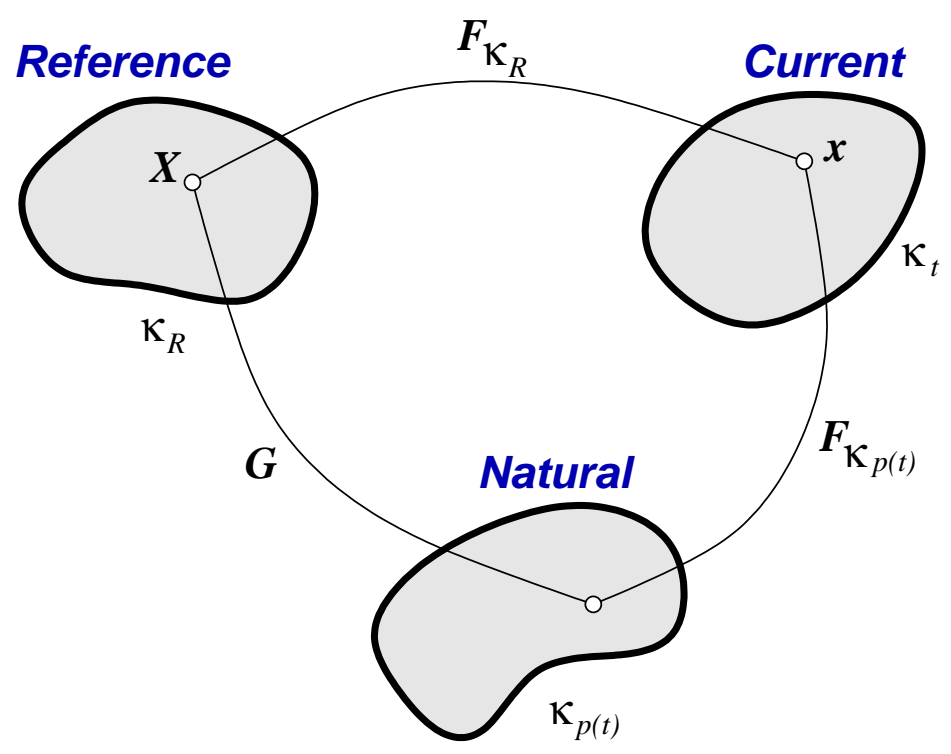

Figure 1: Schematic of the natural configurations of a body $\mathcal{B}$ at time $t$.

where the superscript $T$ denotes transpose. The deformation gradient and the Cauchy-Green stretch tensors, with respect to the natural configuration, are defined by (see Fig. 1)

$$
\boldsymbol{F}_{\kappa_{p(t)}}:=\frac{\partial \chi}{\partial \boldsymbol{X}_{\kappa_{p(t)}}}
$$

and

$$
\boldsymbol{B}_{\kappa_{p(t)}}:=\boldsymbol{F}_{\kappa_{p(t)}} \boldsymbol{F}_{\kappa_{p(t)}^{T}}^{T}, \quad \boldsymbol{C}_{\kappa_{p(t)}}:=\boldsymbol{F}_{\kappa_{p(t)}^{T}}^{T} \boldsymbol{F}_{\kappa_{p(t)}},
$$

respectively. The principal invariants of $\boldsymbol{B}_{\kappa_{p(t)}}$ are given by

$$
\boldsymbol{I}_{\boldsymbol{B}_{\kappa_{p(t)}}}:=\operatorname{tr} \boldsymbol{B}_{\kappa_{p(t)}}, \quad \boldsymbol{I} \boldsymbol{I}_{\boldsymbol{B}_{\kappa_{p(t)}}}:=\frac{1}{2}\left[\left(\operatorname{tr} \boldsymbol{B}_{\kappa_{p(t)}}\right)^{2}-\operatorname{tr} \boldsymbol{B}_{\kappa_{p(t)}}^{2}\right], \quad \boldsymbol{I} \boldsymbol{I} \boldsymbol{I}_{\boldsymbol{B}_{\kappa_{p(t)}}}:=\operatorname{det} \boldsymbol{B}_{\kappa_{p(t)}} .
$$

The mapping $\boldsymbol{G}$, that is a mapping from the reference configuration to the current natural configuration is defined through

$$
\boldsymbol{G}:=\boldsymbol{F}_{\kappa_{p(t)}^{-1}}^{-1} \boldsymbol{F}_{\kappa_{R}}
$$

The velocity gradients $\boldsymbol{L}$ and $\boldsymbol{L}_{\kappa_{p(t)}}$, and their respective symmetric parts $\boldsymbol{D}$ and $\boldsymbol{D}_{\kappa_{p(t)}}$, are defined through

$$
\boldsymbol{L}:=\frac{d}{d t}\left(\boldsymbol{F}_{\kappa_{R}}\right) \boldsymbol{F}_{\kappa_{R}}^{-1}, \quad \boldsymbol{L}_{\kappa_{p(t)}}:=\frac{d \boldsymbol{G}}{d t} \boldsymbol{G}^{-1}
$$




$$
\boldsymbol{D}=: \frac{1}{2}\left(\boldsymbol{L}+\boldsymbol{L}^{T}\right), \quad \boldsymbol{D}_{\kappa_{p(t)}}=: \frac{1}{2}\left(\boldsymbol{L}_{\kappa_{p(t)}}+\boldsymbol{L}_{\kappa_{p(t)}^{T}}^{T}\right) .
$$

where $\frac{d}{d t}$ denotes the usual material time derivative.

The upper-convected Oldroyd derivative of a tensor $\boldsymbol{A}$ is defined by

$$
\stackrel{\nabla}{\boldsymbol{A}}=: \frac{d \boldsymbol{A}}{d t}-\boldsymbol{L} \boldsymbol{A}-\boldsymbol{A} \boldsymbol{L}^{T} .
$$

It then follows that

$$
\stackrel{\nabla}{\boldsymbol{B}}_{\kappa_{p(t)}}=\frac{d \boldsymbol{B}_{\kappa_{p(t)}}}{d t}-\boldsymbol{L} \boldsymbol{B}_{\kappa_{p(t)}}-\boldsymbol{B}_{\kappa_{p(t)}} \boldsymbol{L}^{T}=-2 \boldsymbol{F}_{\kappa_{p(t)}} \boldsymbol{D}_{\kappa_{p(t)}} \boldsymbol{F}_{\kappa_{p(t)}}^{T} .
$$

Since we shall model blood as an incompressible viscoelastic shear-thinning fluid, we shall require that

$$
\operatorname{tr} \boldsymbol{D}=0, \quad \operatorname{tr} \boldsymbol{D}_{\kappa_{p(t)}}=0 .
$$

The above kinematic conditions suffice for our purpose.

\subsection{A shear-thinning viscoelastic rate type fluid model for blood}

For details of the development of rate type viscoelastic fluid models capable of instantaneous elastic response, within the context of a specific Helmholtz potential and rate of dissipation, we refer the reader to [28]. The theory requires the specification of a Helmholtz potential $\psi$ and a rate of dissipation $\xi$ that satisfies

$$
\xi=\boldsymbol{T} . \boldsymbol{D}-\rho \frac{d \psi}{d t}
$$

where $\rho$ denotes the fluid density and $\boldsymbol{T}$ is the stress tensor. The model is assumed to meet the requirement that amongst all processes that are accessible to the body it chooses that which maximizes the rate of dissipation (in general, the rate of entropy production). In this study we are assuming that the processes undergone by blood are isothermal, otherwise we will have to modify equation (2.3).

We shall assume that the specific Helmholtz potential is given by

$$
\psi=\frac{\mu}{2}\left(\boldsymbol{I}_{\boldsymbol{B}_{\kappa_{p}(t)}}-3\right) .
$$

Thus, we are assuming that the fluid (blood) stores energy like a neo-Hookean solid. The constant $\mu$ that appears in the above equation is the shear modulus and it is non-negative (in fact, positive) based on physical considerations.

Next, we assume that the rate of dissipation function is given by

$$
\xi=\alpha\left(\boldsymbol{D}_{\kappa_{p(t)}} . \boldsymbol{B}_{\kappa_{p(t)}} \boldsymbol{D}_{\kappa_{p(t)}}\right)^{\gamma}+\eta_{1} \boldsymbol{D} \cdot \boldsymbol{D} \text {. }
$$


where $\alpha, \gamma$ and $\eta_{1}$ are material parameters. When $\gamma=0$, the above model corresponds to the rate of dissipation in a generalized Oldroyd-B fluid (see [28]) and when $\gamma=1$ and $\eta_{1}=0$, the rate of dissipation corresponds to that of a generalized Maxwell fluid. On the basis of physical considerations, we shall assume that $\alpha$ and $\eta_{1}$ are non-negative.

A lengthy but straightforward procedure based on the maximization of the rate of dissipation leads to (see Anand et al. [3])

$$
\begin{aligned}
& \boldsymbol{T}=-p \mathbf{1}+\boldsymbol{S} \\
& \boldsymbol{S}=\mu \boldsymbol{B}_{\kappa_{p(t)}}+\eta_{1} \boldsymbol{D} \\
& \stackrel{\nabla}{\boldsymbol{B}}_{\kappa_{p(t)}}=-2\left(\frac{\mu}{\alpha}\right)^{1+2 n}\left(\operatorname{tr}\left(\boldsymbol{B}_{\kappa_{p(t)}}\right)-3 \lambda\right)^{n}\left[\boldsymbol{B}_{\kappa_{p(t)}}-\lambda \mathbf{1}\right], \\
& \lambda=\frac{3}{\operatorname{tr}\left(\boldsymbol{B}_{\kappa_{p(t)}^{-1}}^{-1}\right)}, \\
& n=\frac{\gamma-1}{1-2 \gamma}
\end{aligned}
$$

In the above equations, $\stackrel{\nabla}{\boldsymbol{B}}_{\kappa_{p(t)}}$ stands for the upper-convected Oldroyd derivative of tensor $\boldsymbol{B}_{\kappa_{p(t)}}$ defined by (2.2), 1 is the unit (identity) tensor, $p$ denotes the Lagrange multiplier that is a consequence of the requirement of incompressibility and $S$ is usually referred to as the extra stress tensor, or the constitutively determined part of the tensor $\boldsymbol{T}$.

\subsubsection{Governing equations}

Since blood is modeled as an incompressible fluid, all motions have to satisfy the balance of mass

$$
\operatorname{div} \boldsymbol{u}=0
$$

and the balance of linear momentum

$$
\rho \frac{d \boldsymbol{u}}{d t}=\operatorname{div} \boldsymbol{T}
$$

where $\boldsymbol{u}$ is the velocity field and $\rho$ denotes the density of blood. These equations have to be solved simultaneously with the constitutive relations (2.6) - (2.10) wherein the expression for the stress is substituted into (2.12) to obtain the governing equation for the velocity field $\boldsymbol{u}$.

The following parameters obtained from human blood [1] will be used in the numerical simulations of this model:

$$
\eta_{1}=0.01 \mathrm{~Pa} \cdot \mathrm{s} ; \quad \mu=0.1611 \mathrm{~N} / \mathrm{m}^{2} ; \quad n=0.5859 ; \quad K=\left(\frac{\mu}{\alpha}\right)^{1+2 n}=58.0725 \mathrm{~s}^{-1}
$$

( $n$ is positive to ensure shear-thinning behavior). 
Equation (2.8) can be rewritten in a more conventional form in terms of the material timederivative. Let us denote by

$$
\frac{1}{\tau\left(\boldsymbol{B}_{\kappa_{p(t)}}\right)}=2 K\left(\operatorname{tr}\left(\boldsymbol{B}_{\kappa_{p(t)}}\right)-3 \lambda\right)^{n}
$$

where $\tau=\tau\left(\boldsymbol{B}_{\kappa_{p(t)}}\right)$ has the dimension of time and plays a role similar to the relaxation time in the classical Oldroyd-B (Maxwell) model [10]. Using this expression, equation (2.8) for the tensor $\boldsymbol{B}_{\kappa_{p(t)}}$ can be rewritten in the following way

$$
\stackrel{\nabla}{\boldsymbol{B}}_{\kappa_{p(t)}}=-\frac{1}{\tau\left(\boldsymbol{B}_{\kappa_{p(t)}}\right)}\left[\boldsymbol{B}_{\kappa_{p(t)}}-\lambda \boldsymbol{1}\right]
$$

Using the definition of the upper-convected time derivative (2.1), the left-hand side takes the form

$$
\frac{d \boldsymbol{B}_{\kappa_{p(t)}}}{d t}-\left[\boldsymbol{L} \boldsymbol{B}_{\kappa_{p(t)}}+\boldsymbol{B}_{\kappa_{p(t)}} \boldsymbol{L}^{T}\right]=-\frac{1}{\tau\left(\boldsymbol{B}_{\kappa_{p(t)}}\right)}\left[\boldsymbol{B}_{\kappa_{p(t)}}-\lambda \boldsymbol{1}\right]
$$

Finally, expanding the material time-derivative on the left-hand side we end up with

$$
\frac{\partial \boldsymbol{B}_{\kappa_{p(t)}}}{\partial t}+(\boldsymbol{u} \cdot \boldsymbol{\nabla}) \boldsymbol{B}_{\kappa_{p(t)}}=-\frac{1}{\tau}\left[\boldsymbol{B}_{\kappa_{p(t)}}-\lambda \boldsymbol{1}\right]+\left[\boldsymbol{L} \boldsymbol{B}_{\kappa_{p(t)}}+\boldsymbol{B}_{\kappa_{p(t)}} \boldsymbol{L}^{T}\right]
$$

where the coefficients $\lambda$ and $\tau$ are scalar functions of the tensor $\boldsymbol{B}_{\kappa_{p(t)}}$ and its invariants. It is interesting to observe that the classical Oldroyd-B (upper-convected Maxwell) model takes a similar form

$$
\frac{\partial \boldsymbol{T}_{e}}{\partial t}+(\boldsymbol{u} \cdot \nabla) \boldsymbol{T}_{e}=\frac{2 \eta_{e}}{\tau} \boldsymbol{D}-\frac{1}{\tau} \boldsymbol{T}_{e}+\left[\boldsymbol{L} \boldsymbol{T}_{e}+\boldsymbol{T}_{e} \boldsymbol{L}^{T}\right]
$$

$\boldsymbol{T}_{e}$ being the extra stress, $\eta_{e}$ the elastic viscosity coefficient and $\tau$ the (constant) relaxation time.

\section{Numerical simulations}

Some numerical experiments have been performed in order to check the functionality of the above described shear-thinning viscoelastic fluid model for blood flow (further denoted as $b N O B$ ) and compare its predictions with the classical Newtonian $(N S)$ and generalized Oldroyd-B $(G O B)$ fluid models, under different flow conditions. The $G O B$ model used here is obtained from the Oldroyd-B model (2.17) (see [10] for details) replacing the total (constant) viscosity by the shear dependent viscosity given by the generalized Cross function

$$
\frac{\eta(\dot{\gamma})-\eta_{\infty}}{\eta_{0}-\eta_{\infty}}=\frac{1}{\left(1+(\alpha \dot{\gamma})^{b}\right)^{a}}
$$

where $\eta_{0}$ and $\eta_{\infty}$ are the asymptotic viscosities at low and high shear rates and parameters $\alpha, a$ and $b$ are estimated by curve fitting of experimental data. These parameters of the $G O B$ model have 
been taken from [10] (see also [26]), except for the asymptotic viscosities $\eta_{0}$ and $\eta_{\infty}$, that have been adjusted to fit the $b N O B$ 's model parameters, namely $\eta_{0}=0.0736 \mathrm{~Pa} \cdot s$ and $\eta_{\infty}=0.005 \mathrm{~Pa} \cdot s$.

The main goal is to evaluate the hemodynamic and rheological characteristics of this new $b N O B$ model in two simple idealized three-dimensional geometries, based on previous works dealing with similar test cases for simpler models as the generalized Newtonian or the classical and generalized Oldroyd-B models $[10,13,14]$.

\subsection{Numerical method}

The numerical method used to solve the governing equations is based on a spatial finite-volume discretization on structured grids and an explicit Runge-Kutta time-stepping scheme, namely a robust modified Runge-Kutta four-stage method [25]. The computational mesh is structured and consists of hexahedral primary control volumes. To evaluate the viscous numerical fluxes also dual finite volumes with octahedral shape and centered around the primary cell faces are used.

We look for steady solutions by a time-marching approach, which means that the unsteady governing equations are solved with steady boundary conditions and steady solutions are recovered when $t \rightarrow \infty$. An artificial compressibility formulation [33], often used in steady flow simulations, is applied to obtain the pressure and to enforce the divergence-free constraint. The continuity equation (2.11) is modified by adding the pressure time-derivative, properly scaled by the artificial speed of sound c (equal to 1 , for simplicity), as follows:

$$
\frac{1}{\mathbf{c}^{2}} \frac{\partial p}{\partial t}+\operatorname{div} \boldsymbol{u}=0
$$

A pressure stabilization technique has been used in the present simulations (see e. g. [33]) to avoid numerical oscillations in the pressure mainly due to the presence of strong gradients. This technique consists in adding a pressure dissipation term (Laplacian) into the right hand side of the modified continuity equation (3.2) such that

$$
\frac{\partial p}{\partial t}+\mathbf{c}^{2} \operatorname{div} \boldsymbol{u}=\epsilon \Delta p
$$

which, in particular, vanishes if the pressure is a linear function of the space coordinates.

The Reynolds number used in the simulations was quite low (of the order of $10^{2}$ ) and thus no additional stabilization was needed for the flow variables. The details of this approach can be found in earlier papers $[11,12]$ and the references therein.

Numerical tests have been performed in two different geometries. The first computational domain represents a non-symmetric (with respect to the bulk flow direction) cosine-shaped vessel stenosis shown in Fig. 2. The stenosed vessel is three-dimensional, rotationally symmetric, with diameter $D=2 R=6.2 \mathrm{~mm}$ which reduces to its one half in the stenosed region. This leads to a $4: 1$ cross-sectional area reduction and thus to a significant local flow acceleration.

The second computational domain, is a curved $90^{\circ}$ elbow shapped vessel with constant curvature, as shown in Fig. 3. The curved vessel is three-dimensional with circular cross-section of the same size as the above described stenosis. The length of the inflow and outflow straight sections are $L_{\text {in }}=L_{\text {out }}=10 R$. 


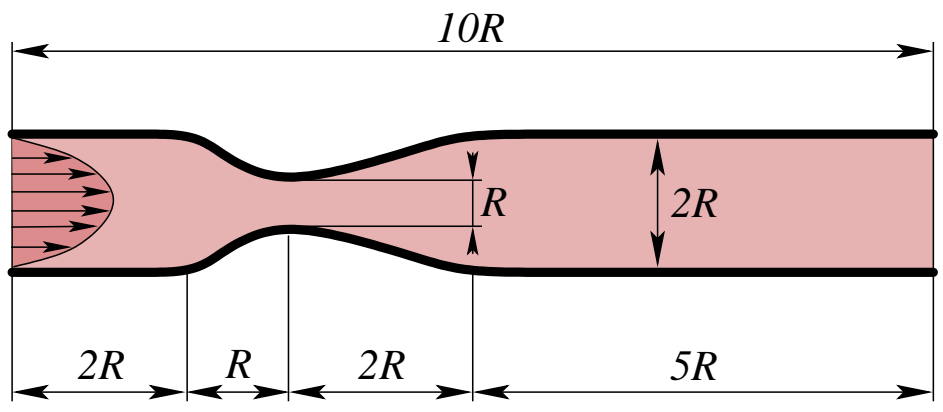

Figure 2: Stenosed vessel

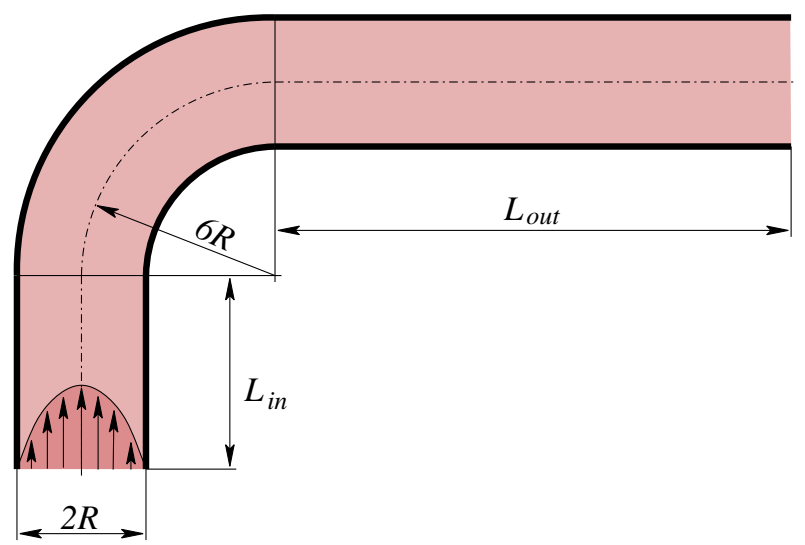

Figure 3: Curved vessel (elbow)

\subsubsection{Computational grid}

The computational domain is discretized using a structured, wall fitted mesh with hexahedral cells and non-uniform axial cell spacing. A multiblock mesh structure was adopted to avoid high distortion of cells, see Fig. 4. The outer mesh block has $64 \times 16 \times 100$ cells, while the central mesh block has $16 \times 16 \times 100$ control volumes. A similar grid structure was used in both test case geometries.

\subsubsection{Boundary conditions}

A parabolic velocity profile with given flow-rate $Q$ is prescribed at the inlet of each domain, for $b N O B, G O B$ and $N S$ models. Homogeneous Neumann conditions for the velocity components are imposed at the outlet and no-slip conditions are prescribed at the wall. Pressure is fixed at the outlet and extrapolated at the other boundaries. Moreover, homogeneous Neumann boundary conditions are prescribed at all boundaries for the components of tensor $\boldsymbol{B}_{\kappa_{p}(t)}$. As an alternative, some simulations have been performed using a Dirichlet type boundary condition $\boldsymbol{B}_{\kappa_{p}(t)}=\mathbf{1}$ prescribed at the inlet, but no significant impact on the solution has been found. 

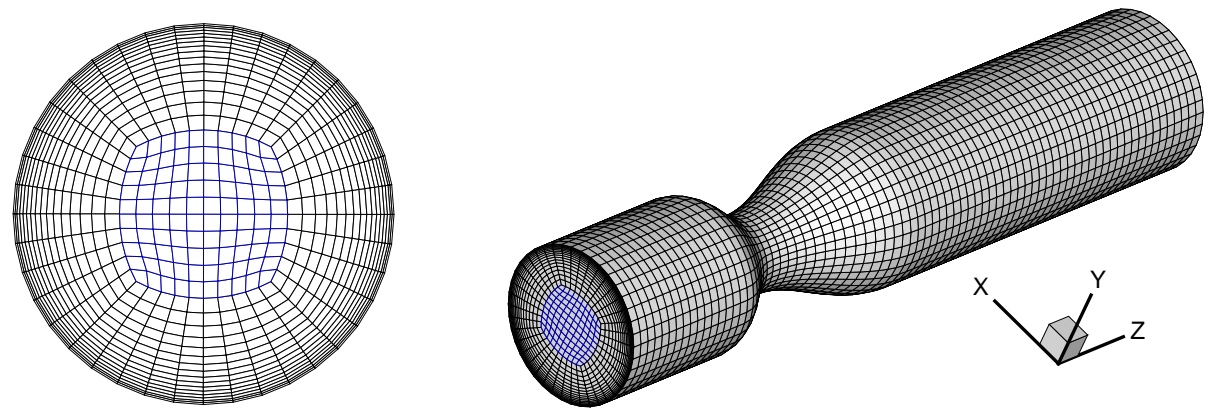

Figure 4: Computational grid structure

\subsection{Numerical Results}

\subsubsection{Stenosed vessel}

The stenosed vessel has been chosen as a test case to study the behavior of the new blood model $b N O B$ in the presence of a high shear region. The sudden (but smooth) reduction of the crosssectional area generates jet-like flow patterns with significant recirculation zones downstream of the stenosis. The sizes of the recirculation zones depend, for a given geometry, mainly on the flow rate and the rheological model. Therefore, three different flow rates $Q=2.0,1.0$ and $0.5 \mathrm{~cm}^{3} / \mathrm{s}$ were investigated in the simulations for the three considered models, using for the $b N O B$ model the blood flow parameters given by (2.13).

Flow field. The influence of the shear-thinning and viscoelastic effects on the qualitative behavior of the flow field can be observed by comparing the results of the axial velocity, radial velocity and pressure contours for both the Newtonian $(N S)$ model (with viscosity $\eta_{1}=2 \eta_{\infty}=0.01 \mathrm{~Pa} \cdot \mathrm{s}$ ) and the shear-thinning viscoelastic fluid model $b N O B$. Figures 5 and 6 show the axial velocity, radial velocity and pressure contours ${ }^{\dagger}$ for the $N S$ and $b N O B$ models, with the flow rates $Q=2.0 \mathrm{~cm}^{3} / \mathrm{s}$ and $Q=0.5 \mathrm{~cm}^{3} / \mathrm{s}$, respectively.

As expected (see previous results in [10] and [14]) there are essentially two main differences between the Newtonian $(N S)$ model solution and the blood model (bNOB) solution.

i) The velocity profile obtained using the $b N O B$ model is flatter in the central part of the vessel, which corresponds to a reduced value of the maximum velocity. As a consequence, the near wall flow is accelerated and thus the recirculation zones become shorter.

ii) The pressure drop is higher ${ }^{\ddagger}$ for the $b N O B$ flow model, due to the local increase of the viscosity (with respect to the Newtonian flow reference viscosity $\eta_{\infty}=\eta_{1} / 2$ ) in the low

\footnotetext{
${ }^{\dagger}$ The color scale, with units in $\mathrm{m} / \mathrm{s}$ (shown below in Fig. 7), is the same in both figures, to allow for a direct comparison. Regions with negative axial velocity (i.e. reversal flow) are plotted in grey color.

${ }_{\ddagger}^{\ddagger}$ The pressure is fixed at the outlet, with the same value for both cases. The resulting higher pressure at the inlet indicates a higher pressure drop.
} 
shear regions, which leads to a higher pressure loss.

Both of these effects can be attributed to the combined shear-thinning and viscoelastic behavior captured by the $b N O B$ model. A better comparison can be done by directly observing figures (7 10) displaying the axial velocity and radial velocity contours for the $N S, G O B$ and $b N O B$ models and both flow rates $Q=2.0 \mathrm{~cm}^{3} / \mathrm{s}$ and $Q=0.5 \mathrm{~cm}^{3} / \mathrm{s}$.
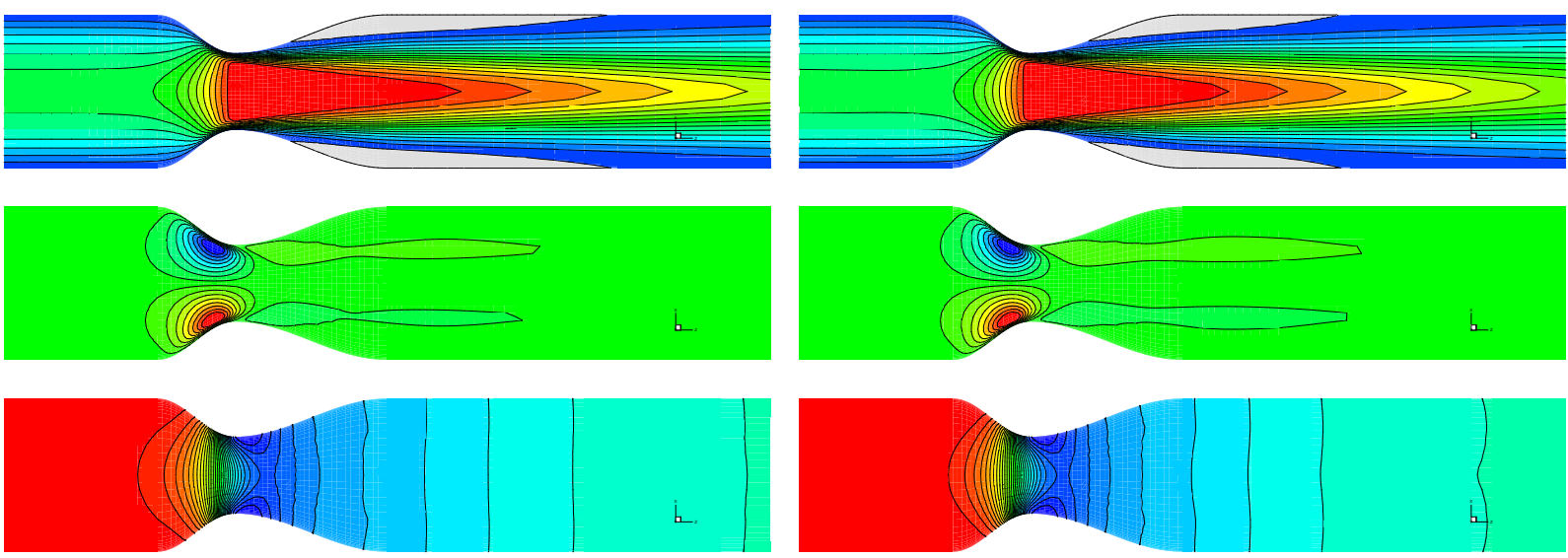

Figure 5: Axial velocity (top), radial velocity (middle) and pressure (bottom) contours, with flow rate $Q=2.0 \mathrm{~cm}^{3} / \mathrm{s}$, for the $N S$ fluid (left) and $b N O B$ fluid (right).
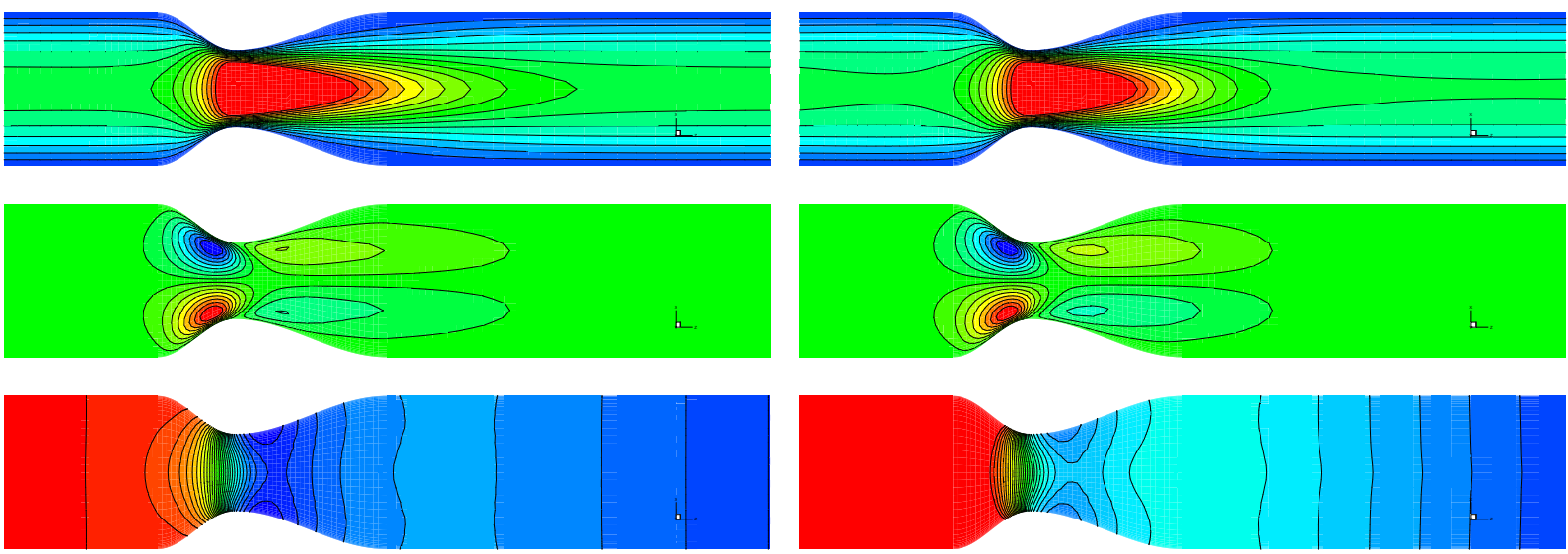

Figure 6: Axial velocity (top), radial velocity (middle) and pressure (bottom) contours, with flow rate $Q=0.5 \mathrm{~cm}^{3} / \mathrm{s}$, for the $N S$ fluid (left) and $b N O B$ fluid (right).

A more detailed study of the rheological effects of the $b N O B$ blood model on the axial velocity contours can be done by directly computing the differences between the $N S$ and the $b N O B$ solutions or between the $G O B$ and $b N O B$ solutions. Figure 11 shows the normalized difference between the $N S$ and the $b N O B$ solutions for different flow rates $Q=2.0,1.0$ and $0.5 \mathrm{~cm}^{3} / \mathrm{s}$. It is obvious that the velocity predicted by the $b N O B$ blood model is lower in the central part of the 

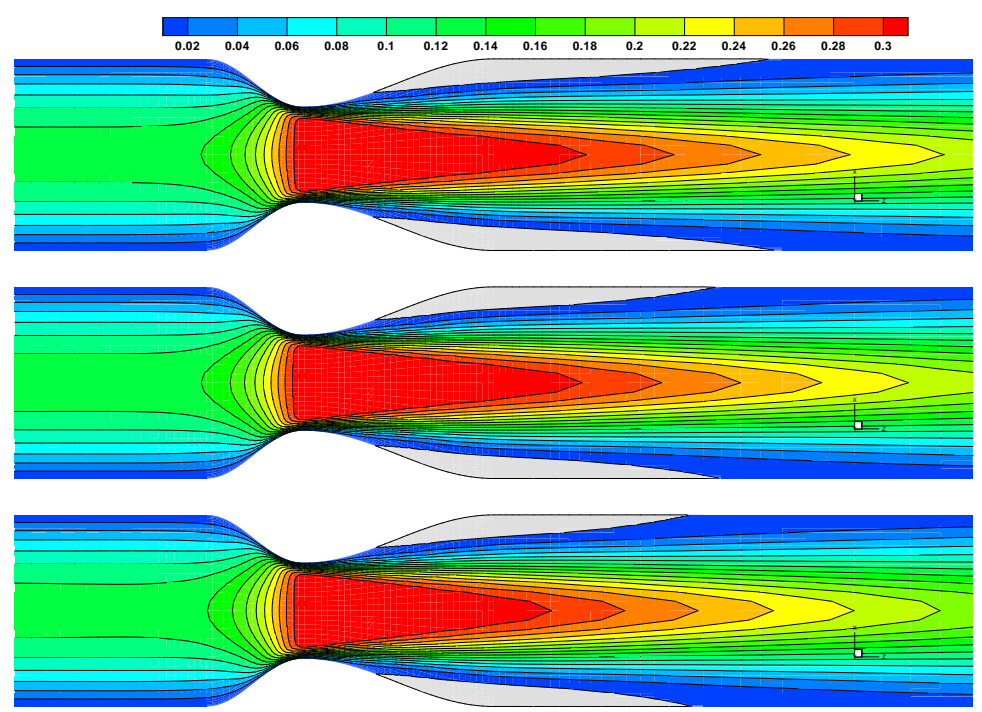

Figure 7: Comparison of the axial velocity contours for $Q=2.0 \mathrm{~cm}^{3} / \mathrm{s}: N S$ fluid (top), $G O B$ model (middle) and $b N O B$ blood model (bottom).

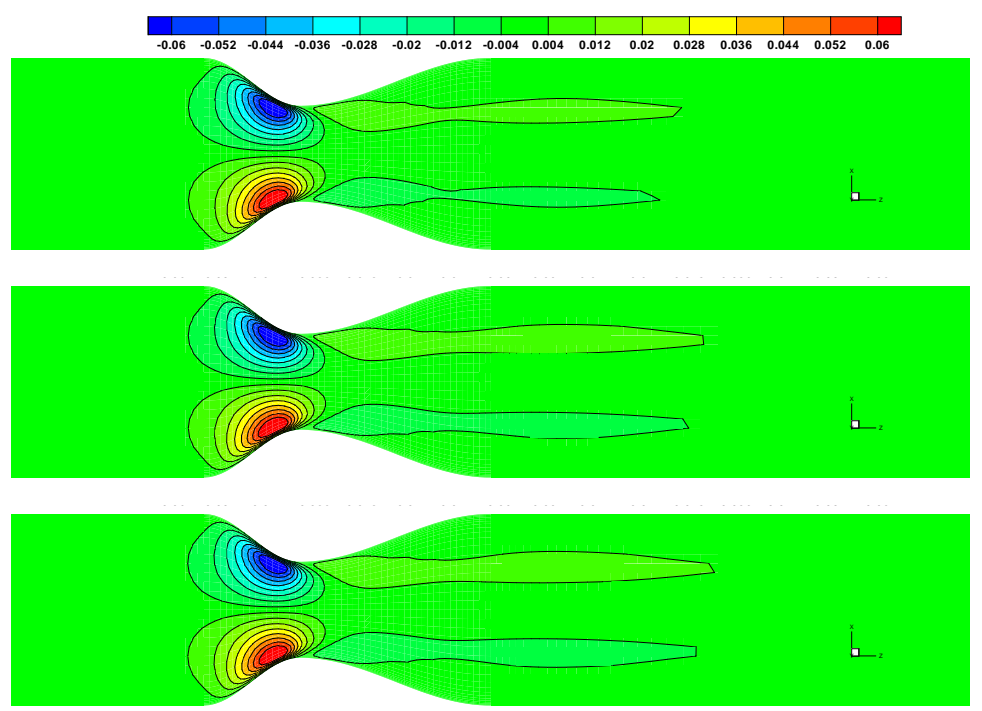

Figure 8: Comparison of the radial velocity contours for $Q=2.0 \mathrm{~cm}^{3} / \mathrm{s}: N S$ fluid (top), $G O B$ model (middle) and $b N O B$ blood model (bottom).

vessel than the one predicted by $N S$. This is compensated by a faster flow in the near-wall region. This marked flattening of the velocity profile is a typical shear-thinning characteristic, not related to the fluid viscoelasticity.

It is also interesting to observe that the magnitude of the axial velocity difference increases with the reduction of the flow rate. This is a natural result, since the Newtonian $(N S)$ solution is computed using a lower viscosity $\eta_{\infty}$ and thus when the flow rate is reduced and, consequently, the 


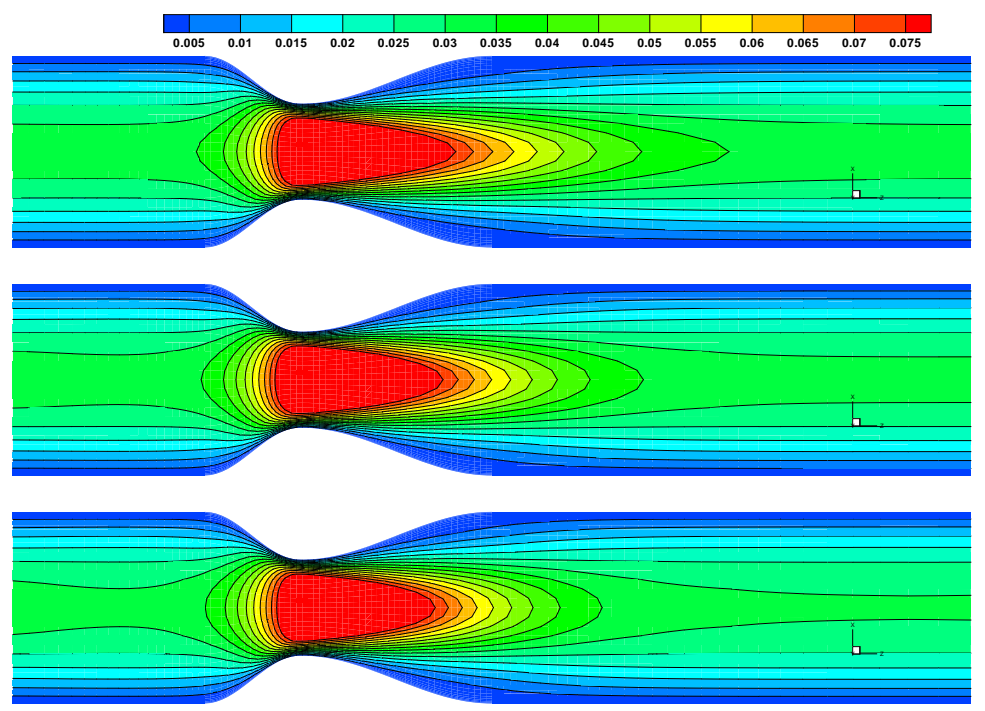

Figure 9: Comparison of the axial velocity contours for $Q=0.5 \mathrm{~cm}^{3} / \mathrm{s}: N S$ fluid (top), $G O B$ model (middle) and $b N O B$ blood model (bottom).

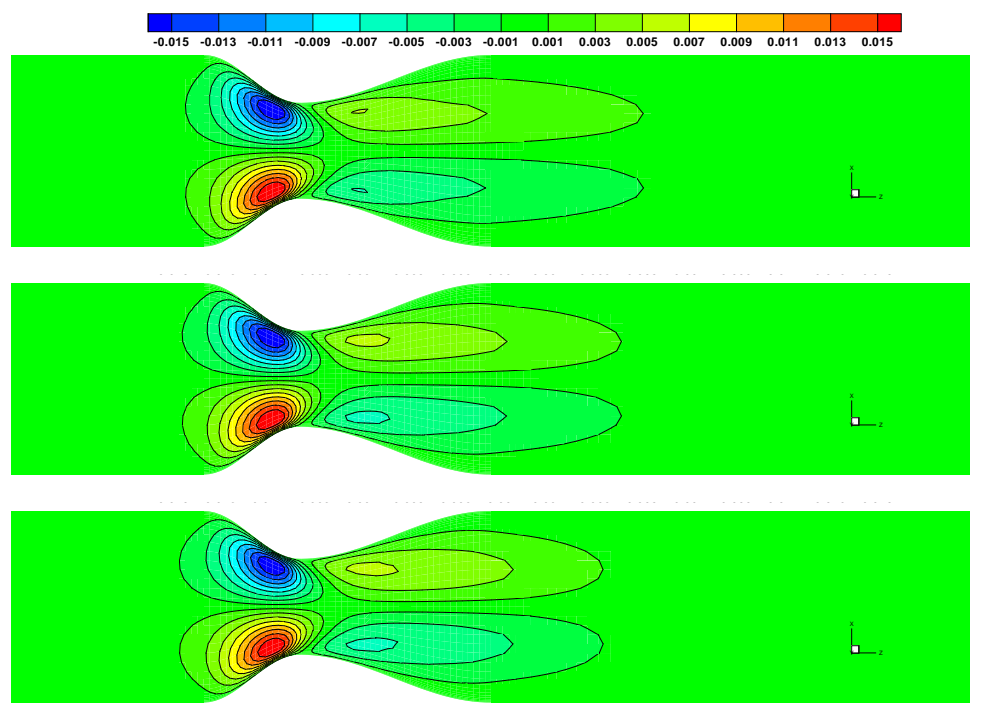

Figure 10: Comparison of the radial velocity contours for $Q=0.5 \mathrm{~cm}^{3} / \mathrm{s}: N S$ fluid (top), $G O B$ model (middle) and $b N O B$ blood model (bottom).

local shear-rate reduces as well, this leads to an increase of the local apparent viscosity. Therefore the magnitude of the axial velocity differences is higher in this case. It is obvious that a different choice of the reference viscosity for the $N S$ model could lead to different results.

The normalized difference between the $b N O B$ and $G O B$ axial velocities, corresponding to the three flow rates $Q=2.0,1.0$ and $0.5 \mathrm{~cm}^{3} / \mathrm{s}$ is shown in Figure 12. As expected, the impact of the viscoelasticity on the flow behavior predicted by the $b N O B$ blood model is less significant than 
the shear-thinning one shown in Figure 11.

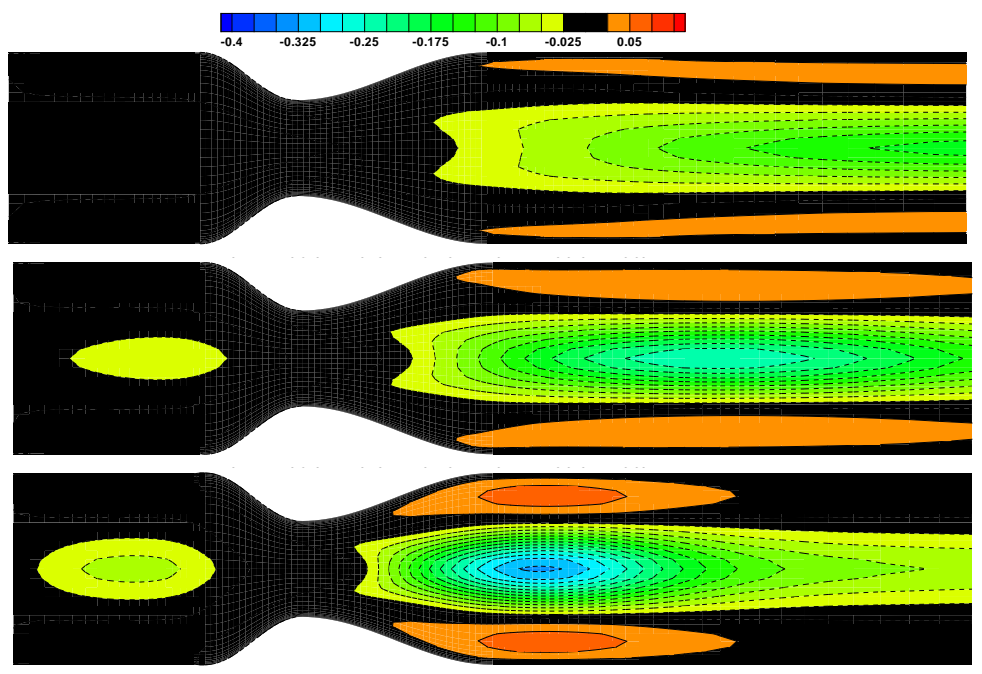

Figure 11: Relative difference of axial velocity $\left(u_{b N O B}-u_{N S}\right) / U_{0}$ for flow rates $Q=$ $2.0,1.0$ and $0.5 \mathrm{~cm}^{3} / \mathrm{s}$ (from top to bottom).

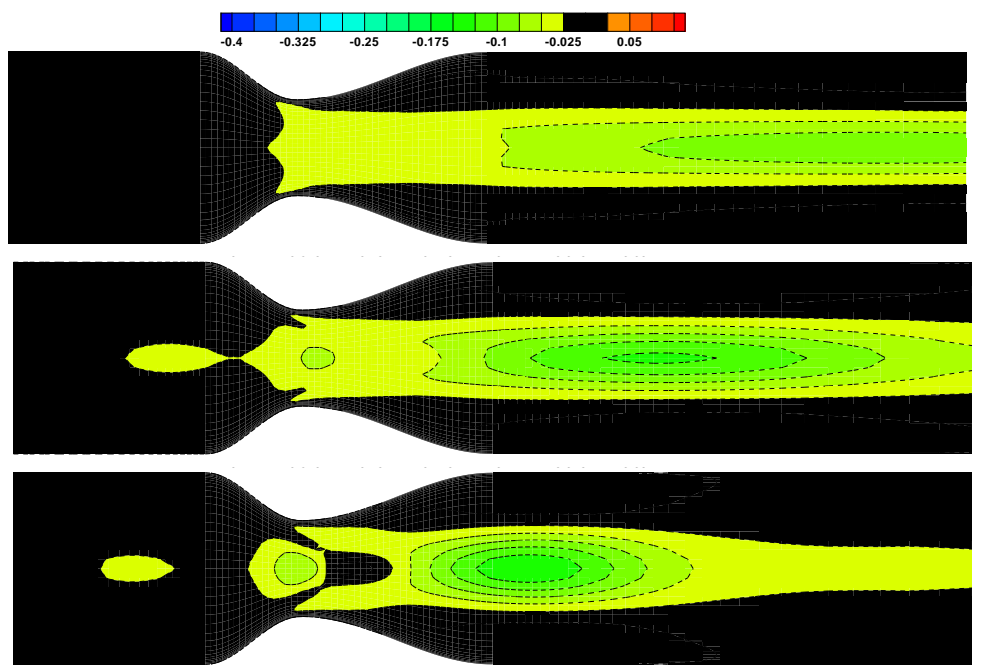

Figure 12: Relative difference of axial velocity $\left(u_{b N O B}-u_{G O B}\right) / U_{0}$ for flow rates $Q=$ 2.0, 1.0 and $0.5 \mathrm{~cm}^{3} / \mathrm{s}$ (from top to bottom).

Forcing effects. The effects of the extra stress can be expressed in terms of the divergence of the tensor $\boldsymbol{B}_{\kappa_{p(t)}}$ The term $\operatorname{div} \boldsymbol{B}_{\kappa_{p(t)}}$ represents an additional force in the balance of linear momentum 
(2.12) related to the viscoelastic behavior predicted by the $b N O B$ blood model. In the present test case the most important component of this force is the axial one. Figure 13 shows the contours of the axial component of $\operatorname{div} \boldsymbol{B}_{\kappa_{p(t)}}$. The blue color represents a force acting against the main flow and causing its deceleration, whose maximum value is attained at the axis of symmetry. This is also a typical behavior captured by shear-thinning models, which has been previously observed in [10] and [14] where the generalized (i.e. shear-thinning) Newtonian and generalized Oldroyd-B models were compared with the classical Newtonian and Oldroyd-B models. Figure 14 shows

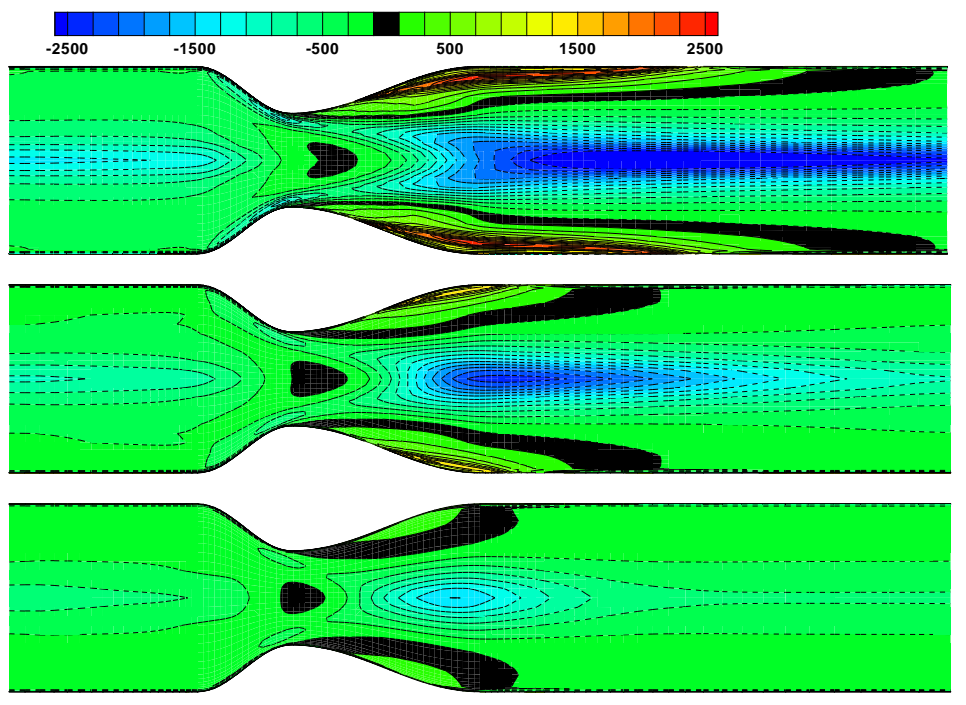

Figure 13: Axial component of the divergence of the tensor $\boldsymbol{B}_{\kappa_{p(t)}}$ for flow rates $Q=2.0,1.0$ and $0.5 \mathrm{~cm}^{3} / \mathrm{s} \quad$ (from top to bottom).

the contours of the six components of the extra stress tensor $\boldsymbol{B}_{\kappa_{p(t)}}$ in the $x-z$ plane, in some cross-sections of the flow domain, for a flow rate $Q=2.0 \mathrm{~cm}^{3} / \mathrm{s}$. The components $b_{i}$ of the tensor $\boldsymbol{B}_{\kappa_{p(t)}}$ are numbered according to the following scheme

$$
\boldsymbol{B}_{\kappa_{p(t)}}=\left(\begin{array}{ccc}
b_{1} & b_{2} & b_{3} \\
b_{2} & b_{4} & b_{5} \\
b_{3} & b_{5} & b_{6}
\end{array}\right)
$$

The contour color scale is the same for all these plots. It is clear that the diagonal components of the tensor $\boldsymbol{B}_{\kappa_{p(t)}}$ are dominant (in magnitude), so the whole tensor is a kind of perturbation of the identity tensor 1 . The same has been observed for the lower flow rates.

\subsection{Curved vessel}

Flows in curved vessels are very challenging and considerably more complex than flows in straight vessels. For inertial Newtonian flows it is well known that a slight curvature of the vessel axis 

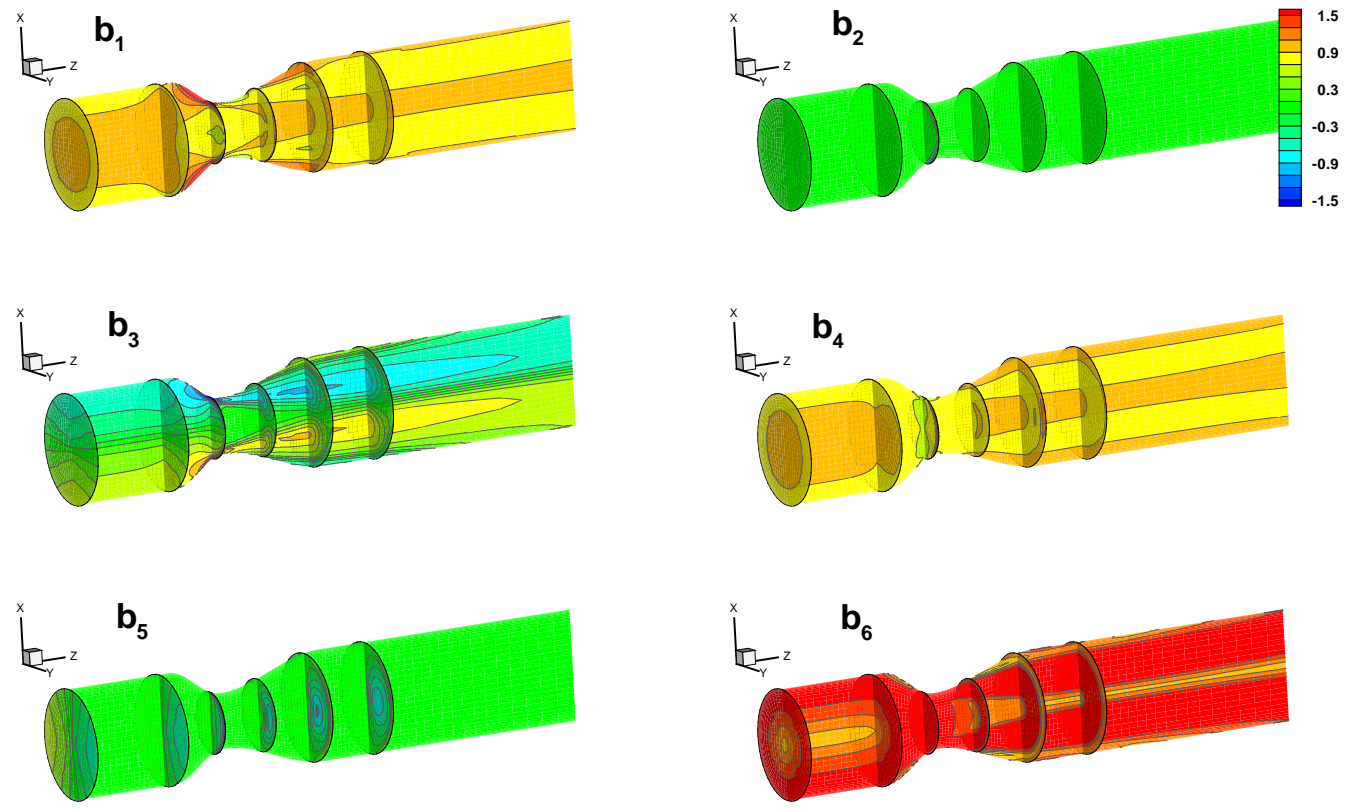

Figure 14: Contours of the components of tensor $\boldsymbol{B}_{\kappa_{p(t)}}$ for the flow rate $Q=2.0 \mathrm{~cm}^{3} / \mathrm{s}$.

induces centrifugal forces on the fluid and, in addition to the primary initial flow, a secondary motion appears, sending fluid outward along the symmetry axis and returning along the upper and lower curved surfaces. This secondary motion is induced by an imbalance between the crossstream pressure gradient and the centrifugal forces developed at the curvature sites, and consists of a pair of symmetrical counter-rotating vortices that is superposed to the axial Poiseuille flow. This results in asymmetrical wall stresses with high shear and low pressure regions (see e.g. [9, 22, 6, 7]).

A $90^{\circ}$ curved vessel (Fig. 3) has been chosen as a second test case to study the behavior of the new blood model $b N O B$ in the presence of high streamline curvature and non-negligible secondary flows. Since the secondary flow pattern is more pronounced at higher flow rates, only the flow rate $Q=2.0 \mathrm{~cm}^{3} / \mathrm{s}$ has been considered for the simulations in this geometry.

One of the important features of the flow predicted by the new blood model $b N O B$ is related to the axial velocity profile. This can clearly be observed in Fig. 15 showing, for the NS, GOB and $b N O B$ models, the plots of the axial velocity in three different sections placed at $30^{\circ}, 60^{\circ}$ and $90^{\circ}$ in the curved part of the vessel. In the case of the $b N O B$ and $G O B$ models flatter axial velocity profiles are obtained when compared to the $N S$ model. As in the case of the flow in the stenosed vessel, this effect can be attributed to the shear-thinning behavior predicted by the $b N O B$ and $G O B$ models where the low shear rates around the centerline of the vessel lead to a local increase of the apparent viscosity. In this case, it is apparent that viscoelasticity of both flows does not play a significant impact in their behavior, however other numerical experiments should be done to prove this ansatz. Curvature effects can be observed in the contours of the axial 
velocity, which are shifted away from the central axis as the curvature of the vessel increases. This is visible in Fig. 16 and, with more details in Fig. 17. Secondary flow streamlines have a similar qualitative behavior for the $N S, G O B$ and $b N O B$ models, as shown in Fig. 16. More details and differences are visible in the comparison of axial and radial velocity contours shown in figures 17 and 18 , where the magnitude of the secondary flow velocities is clearly lower for the $G O B$ and $b N O B$ models due to a higher apparent viscosity.

Figures 19 and 20 show the normalized solution differences between the new $b N O B$ and the classical $N S$ and shear-thinning $G O B$ models. The differences on the axial velocity between the $b N O B$ and the Newtonian model (Fig. 19, left) show the expected slow-down of the core flow (caused by the shear-thinning behavior) in the straight inlet part of the vessel. This is compensated by a faster near-wall flow. As soon as the flow reaches the bended part of the curved vessel, the slow core of the flow is pushed towards the outer wall (see Fig. 20 for a more detailed view), while close to the inner radius of the bend, the flow becomes faster. A similar (although weaker) tendency of the flow behavior can also be seen for the difference between the $b N O B$ and $G O B$ models (Fig. 19, right and Fig. 21). This is mainly due to the fact that both models have a shearthinning viscosity. In the $b N O B$ model shear-thinning effects seem to be slightly more pronounced than in the $G O B$ model. This behavior needs further investigation in a future work.

\section{Conclusions and remarks}

Blood is a very complex multiconstituent material that, in intermediate sized vessels, can be modeled as a single homogeneized continuum which exhibits non-Newtonian behavior. Such response can be captured by the phenomenological shear-thinning viscoelastic constitutive model of Anand and Rajagopal [1]. The present work was devoted to the numerical simulation of this model in two idealized three-dimensional geometries, a stenosis and a curved vessel, to investigate the combined rheological effects of inertia, shear-thinning viscosity and viscoelasticity, for steady flows.

Solutions for the new shear-thinning viscoelastic model $(b N O B)$ have been compared to the classical Navier-Stokes $(N S)$ and generalized Oldroyd-B $(G O B)$ solutions showing, as expected, that the shear-thinning effects are dominant with respect to the viscoelastic ones in both geometries $[10,13,14]$. In the case of the stenosed vessel, the shear-thinning effects are predominant in the recirculation zone downstream the stenosis, increasing as the flow rate (and consequently the shear rate) decreases. In the curved vessel we observed in particular that the axial velocity profile is flatter for the $b N O B$ and $G O B$ models than for the $N S$ model. In summary, we can also conclude that results obtained with the new $b N O B$ model are very similar to those obtained with the classical $G O B$ model, showing in particular that the $b N O B$ model has been properly adjusted and implemented. In addition, this blood rheological model has been created within the novel thermodynamical framework developed by Rajagopal and Srinivasa [28] and the simulations presented here are one of the first making use of that promising approach.

Future work will be devoted to an extension of this numerical study to unsteady flows in geometries like stenosed vessels with different degrees of contraction and curved vessels with different curvatures, to provide a deeper understanding of the significance of the non-Newtonian character- 


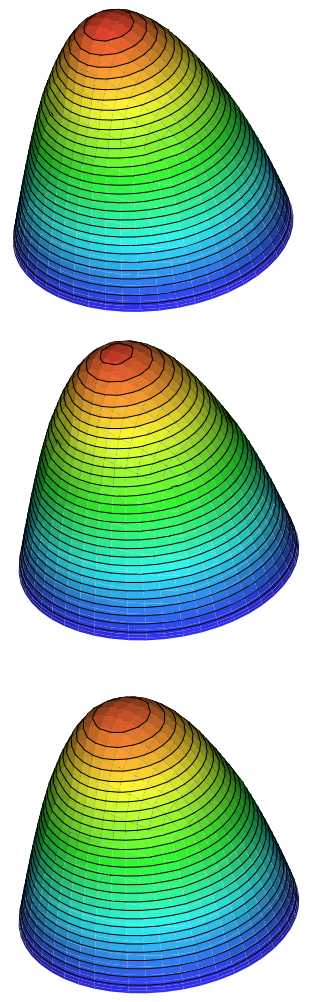

$30^{\circ}$
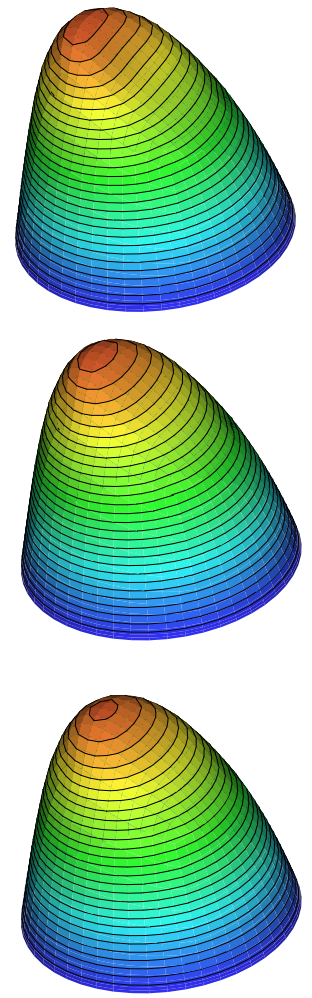

$60^{\circ}$
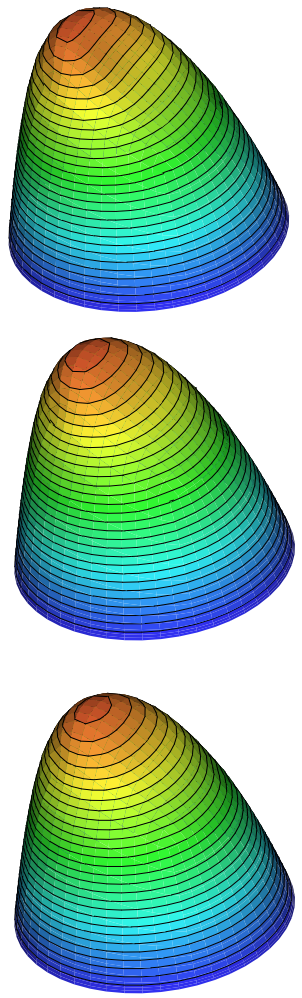

$90^{\circ}$

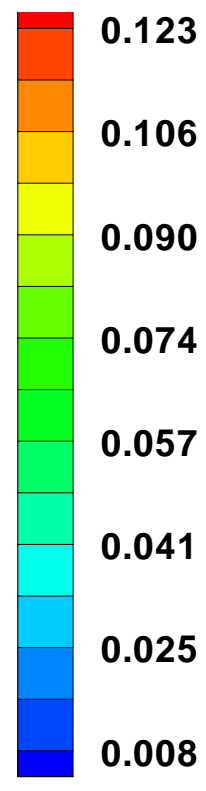

Figure 15: Axial velocity profiles for $N S$ flows (top row), $G O B$ flows (middle row) and $b N O B$ flows (bottom row) in the curved vessel.

istics of blood captured by the $b N O B$ model and their correlation with arterial diseases.

\section{Acknowledgements}

This work has been partially supported by CEMAT/IST through FCT's Funding Program and by the Projects PTDC/MAT/68166/2006 and UT Austin/CA/0047/2008. The first author is grateful for funding provided by the Czech Science Foundation under the Grant No.201/09/0917 and P201/11/1304 and by the Ministry of Education of Czech Republic in the framework of the Research Plan MSM 6840770010.

\section{References}

[1] M. Anand, K.R. Rajagopal. A shear-thinning viscoelastic fluid model for describing the flow of blood. Int. J. of Cardiovascular Medicine and Science, 4, 2 (2004), 59-68. 

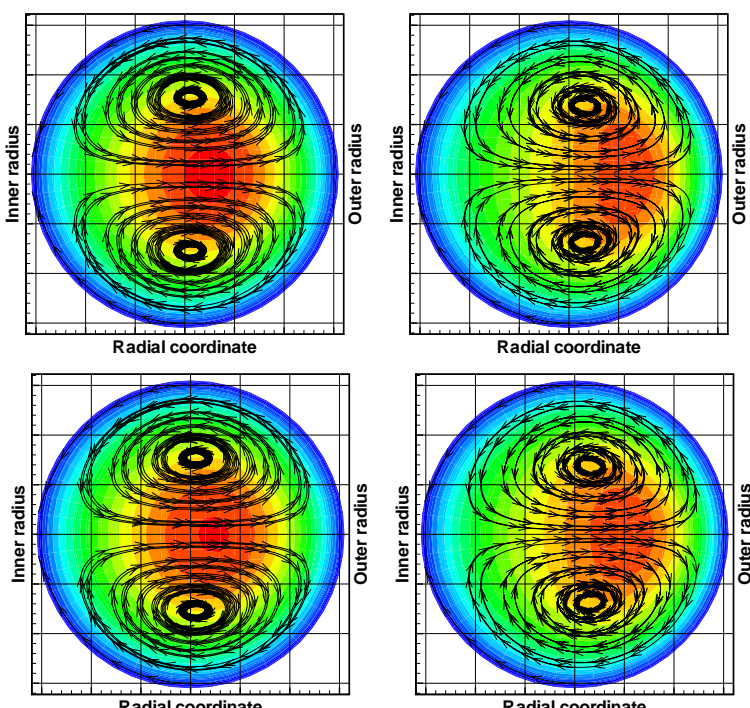

Radial coordinate

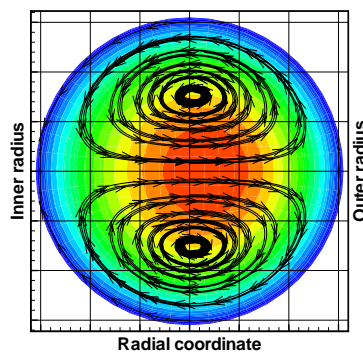

$30^{\circ}$

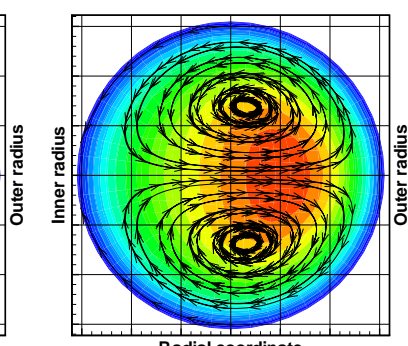

Radial coordinate

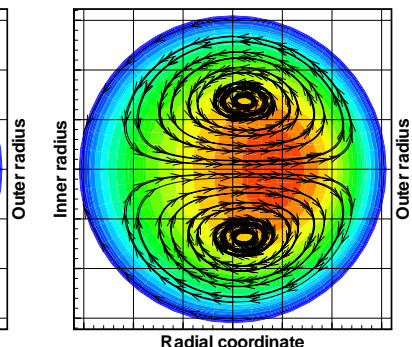

$60^{\circ}$
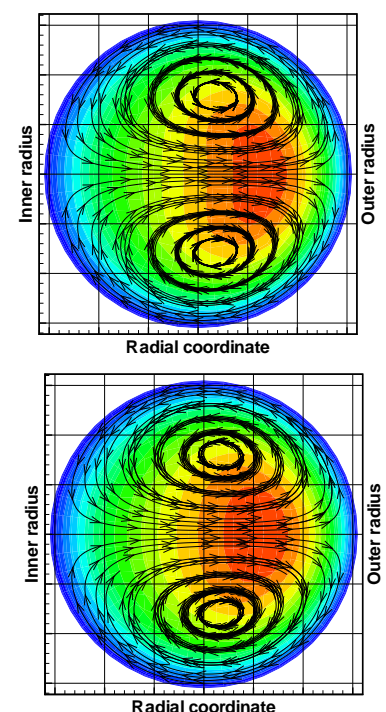

Radial coordinat

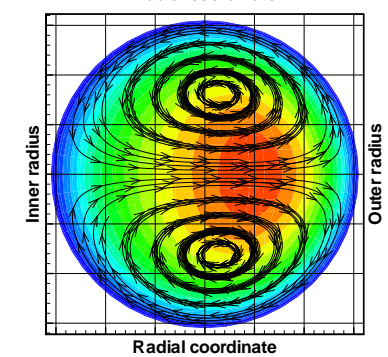

$90^{\circ}$

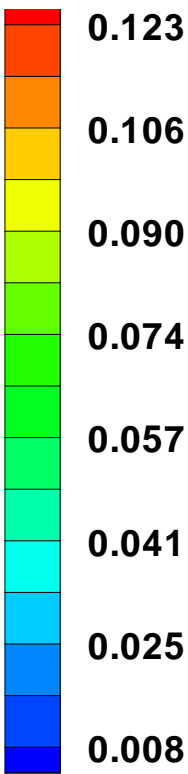

0.008

Figure 16: Axial velocity contours and secondary flow streamlines for the $N S$ (top row), $G O B$ flows (middle row) and the $b N O B$ models (bottom row).

[2] M. Anand, K.R. Rajagopal. A mathematical model to describe the change in the constitutive character of blood due to platelet activation. C. R. Méchanique, 330 (2002), 557-562.

[3] M. Anand, K. Rajagopal, K.R. Rajagopal. A model incorporating some of the mechanical and biochemical factors underlying clot formation and dissolution in flowing blood. J. of Theoretical Medicine, 5, 3-4 (2003), 183-218.

[4] M. Anand, K. Rajagopal, K.R. Rajagopal. A model for the formation and lysis of blood clots. Pathophysiology Haemostasis Thrombosis, 34 (2005), 109-120.

[5] M. Anand, K. Rajagopal, K.R. Rajagopal. A model for the formation, growth, and lysis of clots in quiescent plasma. A comparison between the effects of antithrombin III deficiency and protein C deficiency. J. of Theoretical Biology, 253 (2008), 725-738. 

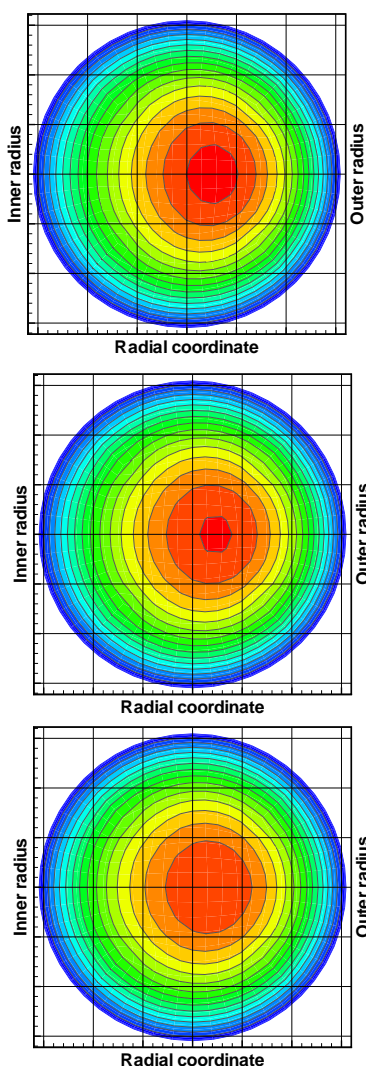

$30^{\circ}$

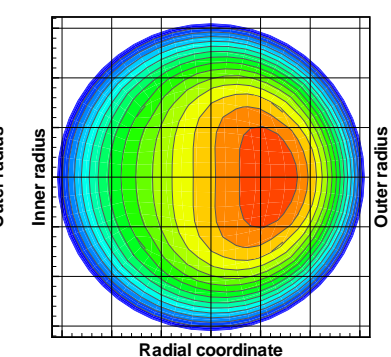

Radial coordinat

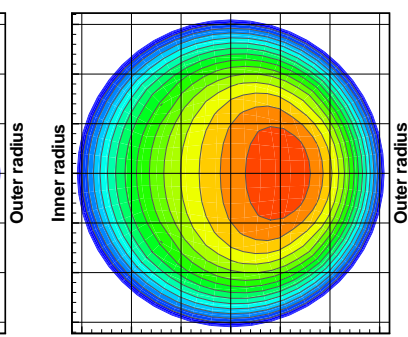

Radial coordinate

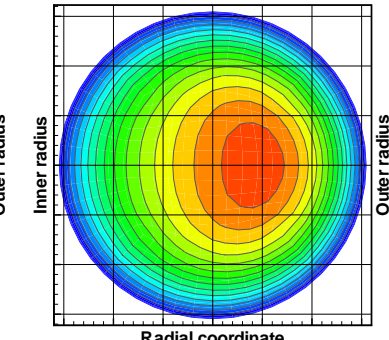

$60^{\circ}$
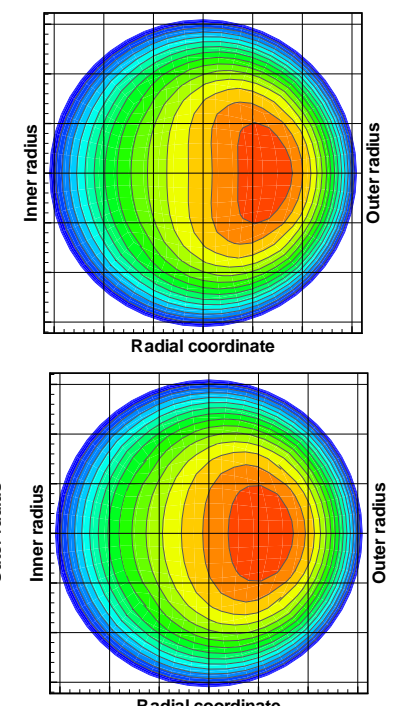

Radial coordinat

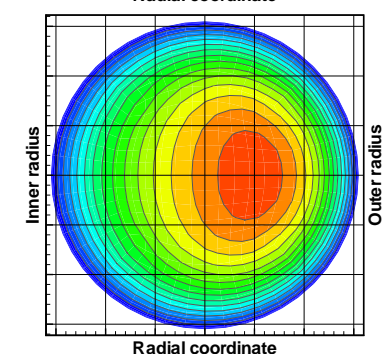

$90^{\circ}$

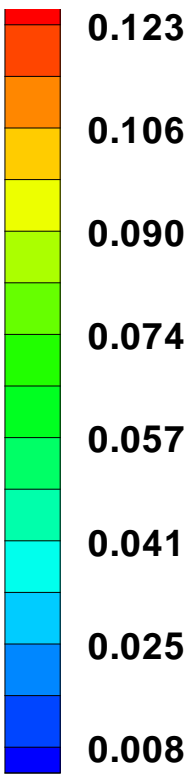

0.008

Figure 17: Axial velocity contours for the $N S$ (top row), $G O B$ flows (middle row) and the $b N O B$ models (bottom row).

[6] N. Arada, M. Pires, A. Sequeira. Viscosity effects on flows of generalized Newtonian fluids through curved pipes. Computers and Mathematics with Applications, 53 (2007), pp. 625646.

[7] N. Arada, M. Pires, A. Sequeira. Numerical simulations of shear-thinning Oldroyd-B fluids in curved pipes. IASME Transactions, Issue 6, 2 (2005), pp. 948-959.

[8] P.D. Bailyk, D.A. Steinman, C.R. Ethier. Simulation of non-Newtonian blood flow in an end-to-side anastomosis. Biorheology, 31 (5) (1994) 565-586.

[9] A.A. Berger, L. Talbot, L.-S. Yao. Flow in curved pipes. Annu. Rev. Fluid Mech., 15 (1983) 461512.

[10] T. Bodnár, A. Sequeira. Numerical Study of the Significance of the Non-Newtonian Nature of Blood in Steady Flow Through a Stenosed Vessel. In: Advances in Mathematical Fluid Mechanics (edited by R. Rannacher \& A. Sequeira), pp. 83-104. Springer Verlag (2010). 

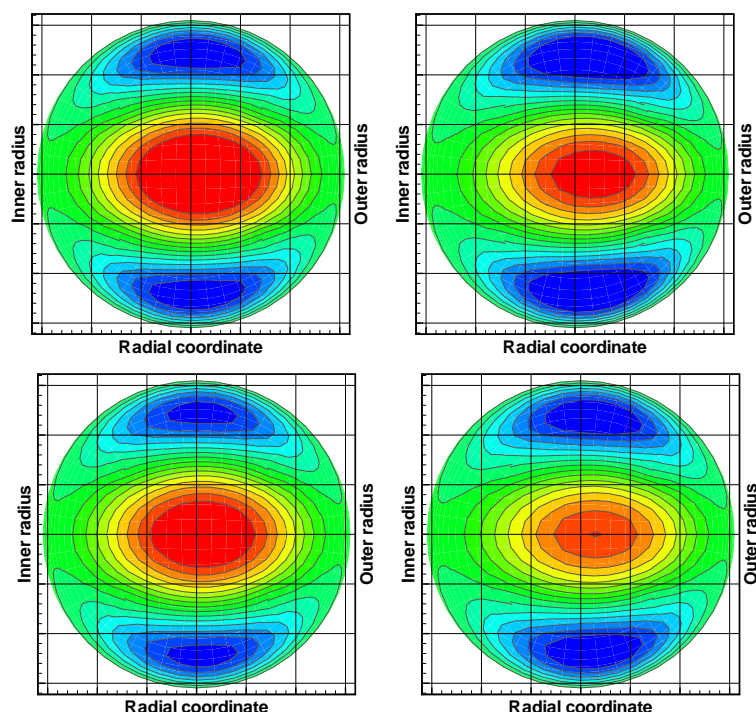

Radial coordinate

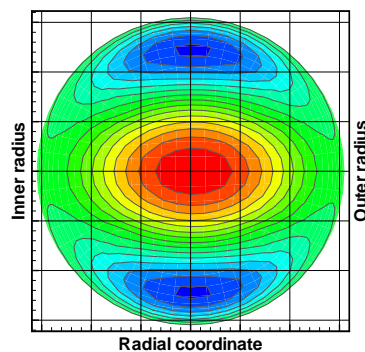

$30^{\circ}$

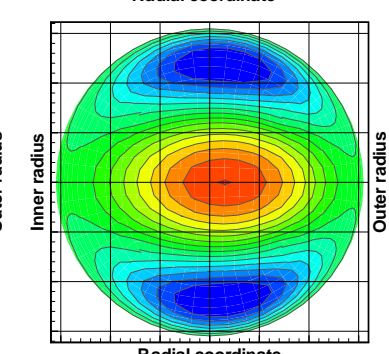

Radial coordinate

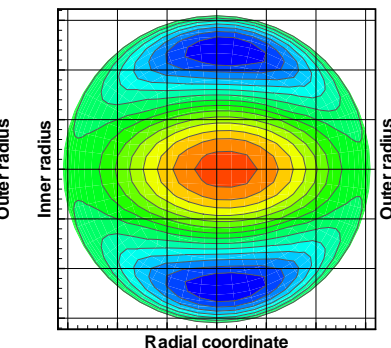

$60^{\circ}$
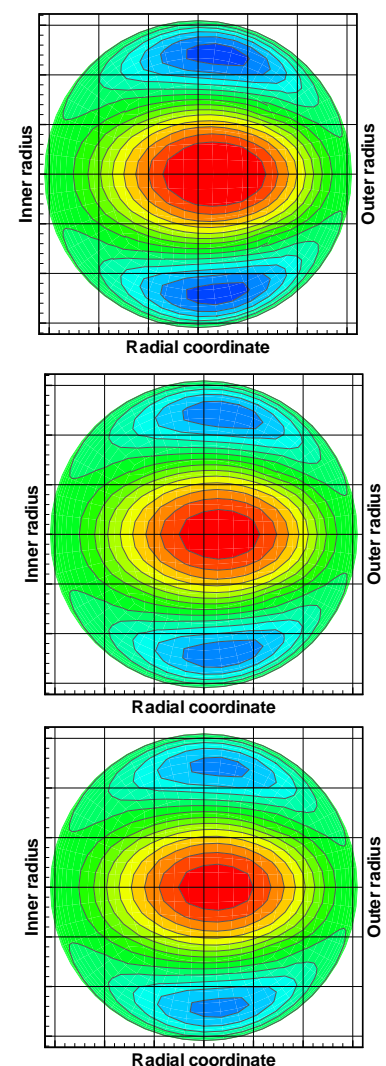

$90^{\circ}$

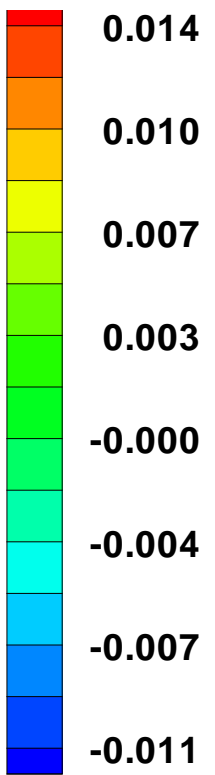

$-0.011$

Figure 18: Radial velocity contours for the $N S$ (top row), $G O B$ flows (middle row) and the $b N O B$ models (bottom row).

[11] T. Bodnár, J. Př́hoda. Numerical simulation of turbulent free-surface flow in curved channel. Journal of Flow, Turbulence and Combustion, 76 (4) (2006) 429-442.

[12] T. Bodnár, A. Sequeira. Numerical simulation of the coagulation dynamics of blood. Computational and Mathematical Methods in Medicine, 9 (2) (2008) 83-104.

[13] T. Bodnár, A. Sequeira, L. Pirkl. Numerical Simulations of Blood Flow in a Stenosed Vessel under Different Flow Rates using a Generalized Oldroyd - B Model In: Numerical Analysis and Applied Mathematics, Vols 1 and 2. Melville, New York: American Institute of Physics, (2009), vol. 2, pp. 645-648.

[14] T. Bodnár, A. Sequeira, M. Prosi. On the Shear-Thinning and Viscoelastic Effects of Blood Flow under Various Flow Rates. Applied Mathematics and Computation, 217 (2011), 50555067. 


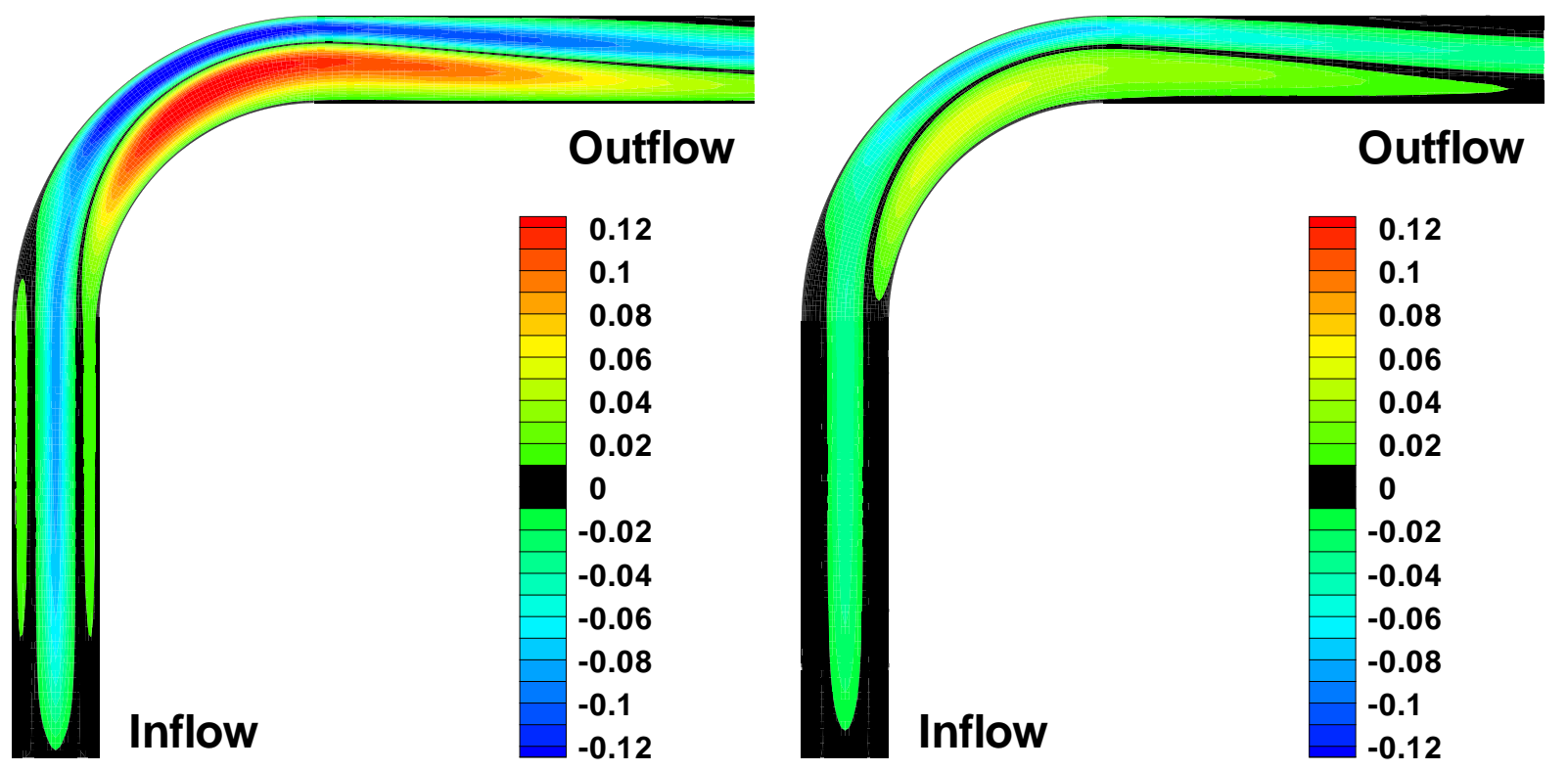

Figure 19: Relative difference of axial velocity $\left(u_{b N O B}-u_{N S}\right) / U_{0}$ (left) and $\left(u_{b N O B}-u_{G O B}\right) / U_{0}$ (right).

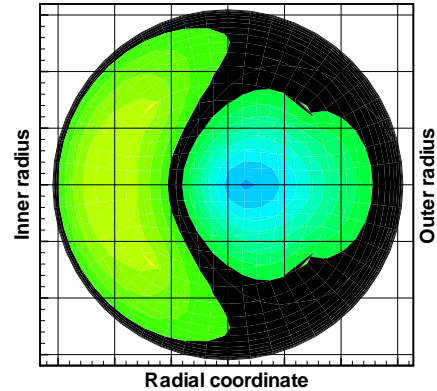

$30^{\circ}$

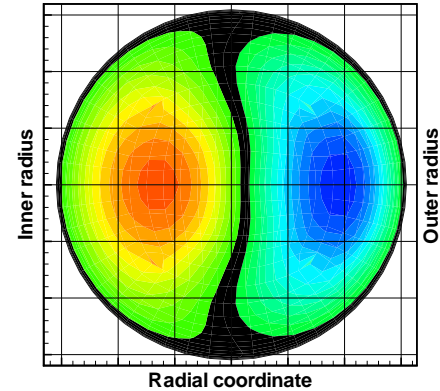

$60^{\circ}$

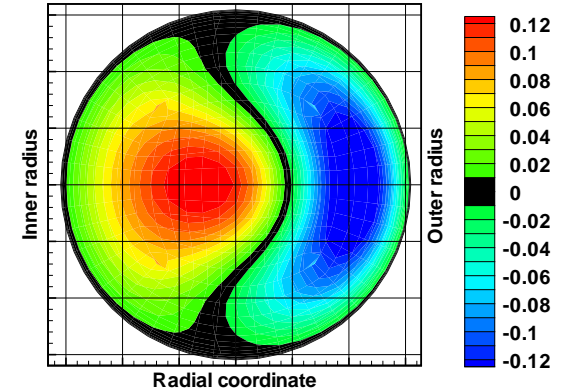

$90^{\circ}$

Figure 20: Relative difference of axial velocity $\left(u_{b N O B}-u_{N S}\right) / U_{0}$.

[15] S.E. Charm, G.S. Kurland. Viscometry of human blood for shear rates of 0-100,000 $\mathrm{sec}^{-1}$. Nature, 206 (1965), 617-618.

[16] S. Chien, S. Usami, H.M. Taylor, J.L. Lundberg, M.I. Gregersen. Effect of hematocrit and plasma proteins on human blood rheology at low shear rates. Journal of Applied Physiology, 21, 1 (1966), 81-87.

[17] S. Chien, S. Usami, R.J. Dellenback, M.I. Gregersen. Blood viscosity: Influence of erythrocyte aggregation. Science, 157, 3790 (1967), 829-831. 


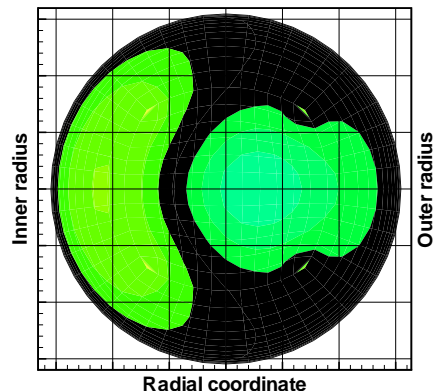

$30^{\circ}$

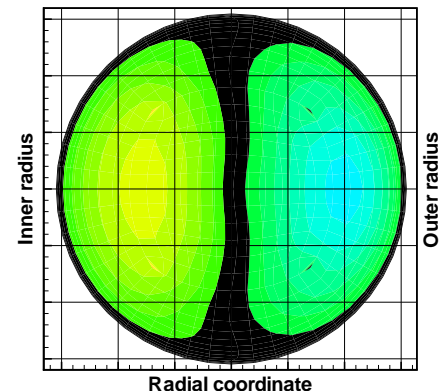

$60^{\circ}$

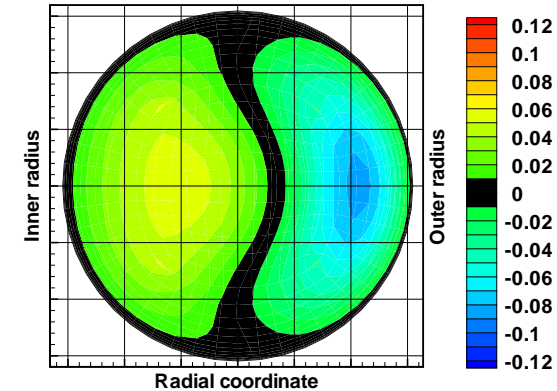

$90^{\circ}$

Figure 21: Relative difference of axial velocity $\left(u_{b N O B}-u_{G O B}\right) / U_{0}$.

[18] S. Chien, S. Usami, R.J. Dellenback, M.I. Gregersen. Blood viscosity: Influence of erythrocyte deformation. Science, 157, 3790 (1967), 827-829.

[19] S. Chien, S. Usami, R. J. Dellenback, M.I. Gregersen. Shear-dependent deformation of erythrocytes in rheology of human blood. American Journal of Physiology, 219 (1970), 136-142.

[20] S. Chien, K.L.P. Sung, R. Skalak, S. Usami, A.L. Tozeren. Theoretical and experimental studies on viscoelastic properties of erythrocyte membrane. Biophysical Journal, 24, 2 (1978), 463-487.

[21] E.A. Evans, R.M. Hochmuth. Membrane viscoelasticity. Biophysical Journal, 16, 1 (1976), $1-11$.

[22] Y. Fan, R.I. Tanner, N. Phan-Thien. Fully developed viscous and viscoelastic flows in curved pipes. J. Fluid Mech., 440 (2001), 327-357.

[23] F. Gijsen, F. van de Vosse, J. Janssen. The influence of the non-Newtonian properties of blood on the flow in large arteries: steady flow in a carotid bifurcation model. Journal of Biomechanics, 32 (1999), 601-608.

[24] J. Hron, J. Málek, S. Turek. A numerical investigation of flows of shear-thinning fluids with applications to blood rheology. Int. J. Numer. Meth., Fluids, 32 (2000), 863-879.

[25] A. Jameson, W. Schmidt, E. Turkel. Numerical solutions of the Euler equations by finite volume methods using Runge-Kutta time-stepping scheme. In:AIAA 14th Fluid and Plasma Dynamics Conference, Palo Alto (1981), AIAA paper 81 - 1259.

[26] A. Leuprecht, K. Perktold. Computer simulation of non-Newtonian effects of blood flow in large arteries. Comp. Methods in Biomech. and Biomech. Eng., 4 (2001), 149-163.

[27] D. Quemada. Rheology of concentrated disperse systems III. General features of the proposed non-Newtonian model. Comparison with experimental data. Rheol. Acta, 17 (1978), 643653. 
[28] K.R. Rajagopal, A.R. Srinivasa. A thermodynamic frame work for rate type fluid models. Journal of Non-Newtonian Fluid Mechanics, 80 (2000), 207-227.

[29] K.R. Rajagopal, A.R. Srinivasa. A Gibbs-potential-based formulation for obtaining the response functions for a class of viscoelastic materials. Proc. R. Soc. A, 467 (2011), 39-58.

[30] G.B. Thurston. Viscoelasticity of human blood. Biophysical Journal, 12 (1972), 1205-1217.

[31] G.B. Thurston. Frequency and shear rate dependence of viscoelasticity of blood. Biorheology, 10, 3 (1973), 375-381.

[32] G.B. Thurston. Non-Newtonian viscosity of human blood: Flow induced changes in microstructure. Biorheology, 31(2), (1994), 179-192.

[33] J. Vierendeels, K. Riemslagh, E. Dick. A multi-grid semi-implicit line-method for viscous incompressible and low-Mach-number flows on high aspect ratio grids. J. Comput. Phys., 154 (1999), 310-344. 